\title{
L'application de la théorie de "phonologie comme comportement humain " à l'analyse de la distribution phonotactique des consonnes et des voyelles dans les mots monosyllabiques du français standard
}

\author{
Igor Dreer ${ }^{*}$
}

Université Ben Gourion du Néguev, P.O. Box 653, 84105 Beer-Sheva, Israël

\begin{abstract}
Résumé. Dans cet article, nous appliquons la théorie de "phonologie comme comportement humain » à l'analyse de la distribution phonotactique des consonnes et des voyelles dans les mots monosyllabiques du français standard, c'est-à-dire de l'occurrence des phonèmes dans certains environnements phonétiques plutôt que dans d'autres. Nous allons montrer que la distribution phonotactique peut être expliquée par le principe de lutte mini-max qui «représente la synthèse des besoins et de l'inertie» [23], c'est-à-dire qui consiste à atteindre le maximum de communication avec le minimum d'effort [30]. En d'autres mots, l'analyse va montrer qu'il existe un rapport direct entre l'effort que les locuteurs font pour contrôler les muscles des organes phonateurs, impliqués dans la production des phonèmes, et la fréquence plus élevée ou plus faible de ces phonèmes dans différentes distributions phonotactiques. D'une part, nous allons montrer quantitativement que les suites de mouvements qui sont plus faciles à articuler et, par conséquent, plus faciles à apprendre à contrôler sont préférées à celles qui exigent une augmentation d'effort et, donc, qui sont plus difficiles à apprendre à contrôler. D'autre part, on augmente un effort pour créer un plus grand nombre de distinctions qui peuvent être clairement perçues dans la communication.
\end{abstract}

\begin{abstract}
The application of the theory of "phonology as human behavior" to the analysis of the phonotactic distribution of consonants and vowels in monosyllabic words of Standard French. In this paper, we apply the theory of "phonology as human behavior" to the analysis of the phonotactic distribution of consonants and vowels in monosyllabic words of Standard French, that is, of the occurrence of phonemes in certain phonetic environments rather than in others. We will show that the phonotactic distribution can be explained by the principle of mini-max struggle that "represents the synthesis of needs and inertia" [23], that is which consists in reaching the maximum communication with minimal effort [30]. In other words, the analysis will show that there is a direct
\end{abstract}

\footnotetext{
*dreer@post.bgu.ac.il
} 


\begin{abstract}
connection between the effort applied by speakers to control the muscles of the phonatory organs, involved in the production of phonemes, and the higher or lower frequency of these phonemes in different phonotactic distributions. On the one hand, we will show quantitatively that combinations of movements that are easier to articulate and, hence, easier to learn to control are favored over combinations requiring more effort and, thus, being more difficult to learn to control. On the other hand, a greater effort is invested to create more distinctions that can be clearly perceived in communication.
\end{abstract}

\title{
1 Introduction
}

«[...] La liberté de lier des espèces phonologiques est limitée par la possibilité de lier les mouvements articulatoires. Pour rendre compte de ce qui se passe dans les groupes [de phonèmes], il y a à établir une phonologie où ceux-ci seraient considérés comme des équations algébriques; un groupe binaire implique un certain nombre d'éléments mécaniques et acoustiques qui se conditionnent réciproquement [...].

Si dans le phénomène de la phonation quelque chose offre un caractère universel qui s'annonce comme supérieur à toutes les diversités locales des phonèmes, c'est sans doute cette mécanique réglée dont il vient d'être question. » [29]

La distribution des phonèmes dans les mots d'une langue ne s'effectue pas au hasard, mais elle obéit à certaines régularités et lois universelles de l'articulation et de la perception des phonèmes. Paraphrasant Saussure (ibid.), nous dirons qu'il devient presque impossible de discuter la question des phonèmes en dehors d'une appréciation exacte des lois qui régissent leur combinaison. Cet article propose une analyse de la distribution phonotactique des consonnes et des voyelles dans les mots monosyllabiques du français standard. L'analyse présentée ici est basée sur la théorie de «la phonologie comme comportement humain » (désormais PCH), sur laquelle on reviendra ci-après, développée par Diver [9] pour l'analyse des groupes consonantiques initiaux en anglais. Diver [10,11] a aussi appliqué ses conclusions à l'analyse des groupes consonantiques et vocaliques en général, et en anglais en particulier. C'est dans cette optique que des recherches ont été menées pour d'autres langues parmi lesquelles l'italien (voir Davis [6]), l'hébreu (voir Tobin [31, 34]), l'urdu (voir Azim [2], Jabeen [19]), le mewati (voir Fatihi [15]), l'espagnol (voir Flores [16]) et le biélorusse (voir Dreer [12]). La théorie a été également appliquée à l'analyse des troubles phonologiques, à la dialectologie et à toutes sortes de problèmes historiques, psycholinguistiques et sociolinguistiques (voir Tobin [31-34]).

\section{Le système phonologique traditionnel du français}

Il n'est pas question ici de reprendre tout le débat concernant le système phonologique du français contemporain. Nous nous bornerons à en indiquer les points les plus saillants. Le français est une langue avec un riche système de voyelles, orales et nasales, et un système de vingt et une consonnes et semi-consonnes (ou semi-voyelles) fondamentales. L'inventaire phonologique du français standard (ou 'français de référence', selon Morin [26]), comme on le présente dans [17], se résume dans le Tableau 1 qui suit. 
Tableau 1. Le système phonologique du français standard

Voyelles orales (et glissantes)

\begin{tabular}{lcclcc}
\hline \multirow{2}{*}{ Orales } & \multicolumn{2}{c}{ Antérieures } & & \multicolumn{2}{c}{ Postérieures } \\
\cline { 2 - 3 } \cline { 5 - 6 } & non-labiales & labiales & & non-labiales & labiales \\
\hline fermées & $\mathrm{i} / \mathrm{j}$ & $\mathrm{y} / \mathrm{y}$ & & & $\mathrm{u} / \mathrm{w}$ \\
mi-fermées & $\mathrm{e}$ & $\varnothing$ & & & $\mathrm{o}$ \\
mi-ouvertes & $\varepsilon$ & $\propto(\partial)$ & & & (a) \\
ouvertes & $\mathrm{a}$ & & & & 0 \\
\hline
\end{tabular}

Voyelles nasales

\begin{tabular}{|c|c|c|c|c|}
\hline \multirow[t]{2}{*}{ Nasales } & \multicolumn{2}{|c|}{ Antérieures } & \multicolumn{2}{|c|}{ Postérieures } \\
\hline & non-labiales & labiales & non-labiales & labiales \\
\hline $\begin{array}{l}\text { fermées } \\
\text { mi-fermées }\end{array}$ & & & & \\
\hline $\begin{array}{l}\text { mi-ouvertes } \\
\text { ouvertes }\end{array}$ & $\tilde{\varepsilon}$ & 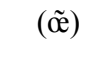 & $\tilde{a}$ & $\tilde{\jmath}$ \\
\hline
\end{tabular}

Consonnes

\begin{tabular}{lcccccccc}
\hline & Bilabiale & $\begin{array}{c}\text { Labio- } \\
\text { dentale }\end{array}$ & Dentale & Alvéolaire & $\begin{array}{c}\text { Alvéo- } \\
\text { palatale }\end{array}$ & Palatale & Vélaire & Uvulaire \\
\hline $\begin{array}{l}\text { occlusives } \\
\text { fricatives }\end{array}$ & $\mathrm{p} / \mathrm{b}$ & $\mathrm{f} / \mathrm{v}$ & $\mathrm{t} / \mathrm{d}$ & $\mathrm{s} / \mathrm{z}$ & $\int / 3$ & & $\mathrm{k} / \mathrm{g}$ & \\
$\begin{array}{l}\text { nasales } \\
\text { latérale }\end{array}$ & $\mathrm{m}$ & & $\mathrm{n}$ & $\mathrm{n}$ & & (n) & ( $1 \mathrm{n})$ & $\mathrm{B}$ \\
\hline
\end{tabular}

Il ressort du Tableau 1 que le voisement est un trait distinctif pour la plupart des consonnes françaises. Par comparaison avec les autres consonnes, la fricative uvulaire

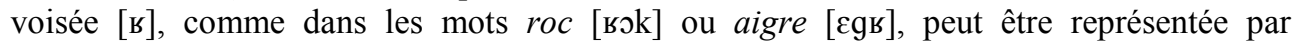
plusieurs variantes combinatoires : l'alvéolaire voisée $[\mathrm{r}]$, l'uvulaire sourde $[\chi]$, la vélaire sourde [x], la vélaire voisée [y], la roulée uvulaire voisée [R] (voir Carton [4], Fagyal et al. [14]). La consonne nasale vélaire [n], que l'on trouvait seulement dans l'exclamation onomatopéique bing [bij], est devenue partie intégrante du français standard assez récemment, avec des emprunts à l'anglais, comme dans le mot ring [bij], ou au chinois, comme dans le nom de la dynastie Ming [min]. Mais à la différence des trois autres consonnes nasales [m, n, n], la [y] n'apparaît qu'en position finale. Quant au système vocalique, le français possède douze voyelles orales et quatre voyelles nasales, dont certaines ont une tendance à l'affaiblissement. En français parisien, la voyelle postérieure [a] tend à être remplacée par son équivalent antérieur [a], et la voyelle labiale nasalisée [ã]

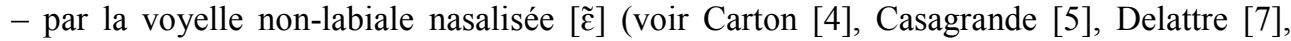
Delbecque [8], Fagyal et al. [14], Walker [38]). La voyelle centrale [ə], également appelée schwa, e muet ou e caduc, fait l'objet de nombreux débats (voir, par exemple, Anderson [1], Hansen [18], Léon [20], Morin [25], Tranel [36], Walker [37]). Certains auteurs pensent que le symbole API [ə] représente une voyelle centrale, plus ou moins arrondie et lâche (voir Walker [38]) qui se prononce souvent comme [œ] (Tranel [35]). D'autres croient que ce phonème peut être confondu soit avec [ø] soit avec [œ] (voir Fagyal et al. [14]).

En nous appuyant sur la $\mathrm{PCH}$, nous montrerons les régularités avec lesquelles les phonèmes sont distribués dans les mots monosyllabiques du français standard. Nous avons inclus dans notre corpus tous les mots monosyllabiques, relevés de la version informatisée du dictionnaire Le Trésor de la langue française (http://atilf.atilf.fr). Selon Davis [6], un dictionnaire comme base de données représente la façon optimale de compter des entrées lexicales indépendamment de contraintes syntactiques, pragmatiques, historiques ou 
stylistiques. Cette approche permet même une évaluation des forces phonologiques dans le mot sans tenir compte de son contenu sémantique (ibid.). En outre, le choix des mots monosyllabiques permet de réduire l'influence de facteurs concomitants (tels que l'accent et la réduction des voyelles dans des syllabes non accentuées) sur la distribution des phonèmes.

Certains sons ou groupes complexes du français ne peuvent apparaître qu'en raison du contact des mots entre eux dans la chaîne parlée, c'est-à-dire que leur apparition n'est pas tout à fait due à des contraintes phonologiques. Comme nous cherchons à établir la nature de contraintes phonologiques sur la distribution des phonèmes au niveau de la langue, et non de la parole, nous nous bornerons à présenter celles qui se placent à l'intérieur des limites morphologiques de mots monosyllabiques, et non au-delà d'elles. Notre échantillon est constitué de 5280 mots monosyllabiques et leurs modifications, prélevés au hasard. Par exemple, l'infinitif cuire [kцi:s], le présent cuis [kui], le subjunctif cuise [kцi:z], les participes passés au masculin cuit [kui] et au féminin cuite [kuit] constituent cinq entrées dans cet échantillon.

\section{Les principes de la PCH}

La PCH se fonde sur la distinction saussurienne entre la langue, un code collectif, et la parole, des messages verbaux individuels (voir Saussure [29]), manifestée par la distribution des phonèmes, c'est-à-dire par leur occurrence dans certains environnements phonétiques plutôt que dans d'autres. Par conséquent, il faut d'abord définir la langue et, sur cette base, essayer d'expliquer cette distribution.

La PCH part du point de vue que la langue est un instrument symbolique, un système de signes saussuriens dont la structure est déterminée tant par sa fonction communicative que par les caractéristiques psychologiques de ses locuteurs (voir Dreer [13]). Il en résulte que dans les analyses phonologiques la $\mathrm{PCH}$ tient compte du facteur communicatif et du facteur humain, indispensables à la compréhension de la structure de la langue aussi bien que de son fonctionnement. Le facteur communicatif conditionne la délimitation des signes de la langue dans la chaîne parlée à partir des phonèmes qui constituent des unités pertinentes minimales à valeur linguistique. En d'autres termes, les phonèmes créent des oppositions distinctives qui correspondent à des différences de valeurs. Le fait qu'on se serve des phonèmes pour articuler les mots et pour les distinguer les uns des autres rend les phonèmes essentiels à la communication. Le facteur humain reflète les particularités psychologiques des locuteurs qui cherchent régulièrement à parvenir à la communication maximale avec le moindre effort. Ce facteur se manifeste par l'intelligence des locuteurs qui tirent des conclusions abstraites des moindres signaux concrets, par l'efficacité des locuteurs qui sont souvent intéressés à investir le moindre effort pour obtenir les meilleurs résultats et par la mémoire des locuteurs, prodigieuse et en même temps restreinte, qui influence directement leur intelligence et efficacité. ${ }^{1}$ La façon dont les phonèmes sont articulés et perçus est également importante pour la compréhension de leur distribution, ce qui entraîne Diver [9] à introduire deux facteurs supplémentaires, à savoir la physiologie de l'appareil phonatoire, impliqué dans le contrôle des muscles des organes phonatoires lors de la production des sons, et les moyens acoustiques qui, d'après Diver, sont impliqués dans la perception des sons par les locuteurs.

De ce qui précède, Diver (ibid.) conclut que les locuteurs apprennent certaines unités distinctives, les phonèmes, qu'ils emploient à des fins communicatives. Nous pouvons juger si la fréquence des phonèmes ou de leurs combinaisons est élevée ou bien faible par les écarts phonotactiques observables, des écarts qui s'étaient formés au cours de l'histoire de la langue et qui ne sont pas particuliers à une langue donnée, mais qui ont un caractère

\footnotetext{
${ }^{1}$ Pour une discussion plus détaillée à ce sujet, nous renvoyons à Tobin [30-31].
} 
universel. On peut analyser ces écarts par rapport au comportement humain en dehors du domaine linguistique. Partant de l'idée qu'un écart significatif représente une difficulté dans un processus d'apprentissage du langage et ayant réalisé ce qui constitue cette difficulté d'apprentissage, on peut identifier les traits distinctifs des phonèmes et essayer de trouver une explication à leur distribution phonotactique.

Nous inspirant de ces principes, nous montrerons que la fréquence relative des phonèmes dans les mots monosyllabiques du français standard peut servir d'exemple de distribution asymétrique. Étant donné que les phonèmes sont produits par le contrôle des muscles des organes phonateurs, suivant Diver ( $\mathrm{ibid}$.), nous émettons l'hypothèse qu'il y a un rapport entre l'effort que les locuteurs font pour contrôler ces muscles et la distribution phonotactique favorisée ou défavorisée des phonèmes qu'ils produisent. Nous visons à montrer que les mouvements musculaires et, donc, les phonèmes qui exigent moins d'effort et qui, par conséquent, sont plus faciles à apprendre à contrôler sont préférés à ceux qui nécessitent une augmentation d'effort et qui sont plus difficiles à apprendre à contrôler. En revanche, on augmente un effort en contrôlant les muscles des organes phonateurs pour créer un plus grand nombre de distinctions qui peuvent être clairement perçues dans des contextes pertinents.

\section{L'analyse}

Pour notre analyse, nous adopterons une classification qui diffère de la classification traditionnelle des phonèmes du français standard et qui repose sur les degrés d'aperture, proposés par Saussure [29]. Comme lui, nous les situerons entre l'occlusion complète, désignée par le chiffre $0^{\circ}$ et l'aperture maximale, désignée par $5^{\circ}$. Cela créera une hiérarchie unique de phonèmes dans laquelle nous désignerons les «consonnes » traditionnelles comme les phonèmes d'occlusion, et les «voyelles" traditionnelles comme les phonèmes d'aperture. En outre, nous suivrons la classification supplémentaire par type de l'écoulement de l'air, proposée par Davis [6], selon laquelle les phonèmes se répartissent entre deux limites extrêmes : du blocage complet de l'écoulement de l'air à l'écoulement libre. Les phonèmes du français standard peuvent être donc classés comme suit :

1. Les consonnes « plosives » ou « occlusives » traditionnelles $([\mathrm{p}],[\mathrm{t}],[\mathrm{k}],[\mathrm{b}],[\mathrm{d}],[\mathrm{g}])$ ont un degré d'aperture zéro parce qu'elles sont produites par une occlusion complète et par un blocage complet de l'écoulement de l'air. La production des phonèmes [m], [n], [n], [n] avec un degré d'aperture zéro, appelés «consonnes nasales » en terminologie phonétique traditionnelle, implique la fermerture de la cavité buccale par l'abaissement du voile du palais ce qui crée un écoulement de l'air libre et nonturbulent par la cavité nasale. Du fait que dans ce cas l'air non-turbulent continue de s'écouler, malgré l'occlusion orale complète, les nasales ressemblent aux phonèmes d'aperture («voyelles », en terminologie traditionnelle). Par conséquent, elles sont situées plus bas dans notre hiérarchie que les « occlusives ».

2. Les consonnes « fricatives » traditionnelles ([f], [v], [s], [z], [S], [3], [ $\mathrm{b}]$ ) ont un degré d'aperture $1^{\circ}$ car leur production n'implique pas l'occlusion complète de la cavité buccale, contrairement aux « occlusives », ce qui crée un écoulement turbulent.

3. Au-delà des nasales, les phonèmes d'occlusion [1], [j], [w], [ $\mathrm{Y}]$ permettent à l'air de s'écouler d'une manière non-turbulente, comme les phonèmes d'aperture le font, bien qu'ils se produisent par une occlusion partielle, avec un degré d'aperture $2^{\circ}$. Dans le cas de la «latérale » [1], le dos de la langue touche la crête alvéolaire, en laissant passer l'air de chaque côté de la langue, sans créer une turbulence dans l'écoulement. Dans le cas des «semi-voyelles » [j], [w], [u], cette occlusion est tellement modérée qu'elle cause à peine une turbulence. D'autre part, les trois phonèmes d'aperture [i], $[\mathrm{y}],[\mathrm{u}]$ ont une fermeture, comparable au degré d'aperture de la «latérale» ou des «semi-voyelles», avec cette seule différence que la 
production des phonèmes d'aperture ne s'accompagne pas de turbulence de l'écoulement de l'air'.

4. Les phonèmes d'aperture $[\mathrm{e}],[\varnothing],[\ni],[\mathrm{o}],[\varepsilon],[\propto],[\circ]$, dits « voyelles orales », avec

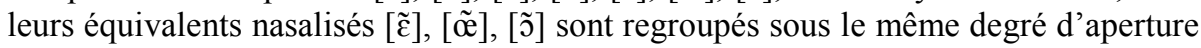
entre $3^{\circ}$ et $4^{\circ}$, remplaçant des catégories traditionnelles des voyelles «mi-fermées » et « mi-ouvertes ».

5. Le phonème central non arrondi $[\mathrm{a}]$, le phonème postérieur $[\mathrm{a}]$ et le phonème nasalisé [ã] ont l'aperture maximale de $5^{\circ}$.

Dans notre classification, nous avons attaché moins d'importantance aux points d'articulation et aux modes d'articulation des phonèmes, figurant dans le Tableau 1. Ce qui est plus important, c'est d'indiquer la façon dont les phonèmes sont articulés, c'est-à-dire « les organes actifs dans l'articulation » (Saussure [29]) plutôt que « les parties passives » (ibid., voir aussi Diver [9]). Sans cette connaissance, nous ne pouvons comprendre ce qui contribue à la production de phonèmes et expliquer leur distribution phonotactique. La langue (et notamment la pointe, la lame et le dos de la langue) et la lèvre inférieure sont les deux organes articulateurs mobiles qui permettent d'obtenir une variation phonologique maximale. Nous avons reclassé les phonèmes du français standard à partir des articulateurs mobiles, des degrés d'aperture des phonèmes et du type de l'écoulement de l'air. Le Tableau 2 présente l'inventaire phonologique révisé du français standard.

Tableau 2. Les phonèmes du français standard, caractérisés par les articulateurs mobiles, les degrés d'aperture des phonèmes et le type de l'écoulement de l'air

\begin{tabular}{|c|c|c|c|c|c|c|c|c|}
\hline \multirow{7}{*}{ 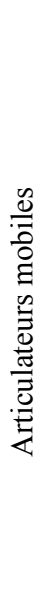 } & Aperture & & $0^{\circ}$ & $0^{\circ}$ & $1^{\circ}$ & $2^{\circ}$ & $3^{\circ}-4^{\circ}$ & $5^{\circ}$ \\
\hline & Lèvres & $\begin{array}{l}0 \\
\mathrm{~L} \\
\mathrm{~V}\end{array}$ & $\begin{array}{l}\mathbf{p} \\
\mathbf{b}\end{array}$ & $\mathbf{m}$ & $\begin{array}{l}\mathbf{f} \\
\mathbf{v}\end{array}$ & & & \\
\hline & Pointe (Apex) & $\begin{array}{l}0 \\
\mathrm{~L} \\
\mathrm{~V}\end{array}$ & $\begin{array}{l}\mathbf{t} \\
\mathbf{d}\end{array}$ & $\mathbf{n}$ & $\begin{array}{l}\mathbf{S} \\
\mathbf{z}\end{array}$ & 1 & & \\
\hline & Lèvres + Lame & $\mathrm{L}$ & & & & у 4 & $\mathbf{E}$ & \\
\hline & Lame & $\begin{array}{l}0 \\
\mathrm{~L} \\
\mathrm{~V}\end{array}$ & & $\mathbf{n}$ & $\begin{array}{l}\int \\
3\end{array}$ & $\mathbf{j} \mathbf{i}$ & $\begin{array}{c}\mathbf{E} \\
\tilde{\mathbf{E}}\end{array}$ & $\mathbf{A}$ \\
\hline & Lèvres + Dos & $\begin{array}{l}\mathrm{L} \\
\mathrm{V}\end{array}$ & & & & $\mathbf{w} \mathbf{u}$ & $\begin{array}{l}\mathbf{O} \\
\tilde{\mathbf{j}}\end{array}$ & \\
\hline & Dos & $\begin{array}{l}0 \\
\mathrm{~L} \\
\mathrm{~V}\end{array}$ & $\begin{array}{l}\mathbf{k} \\
\mathbf{g}\end{array}$ & n & $\mathbf{6}$ & & & $\tilde{\mathbf{a}}$ \\
\hline
\end{tabular}

Ce tableau présente non seulement les organes mobiles, la lèvre inférieure et les trois parties de la langue (la pointe, la lame et le dos), au moyen desquels les phonèmes sont articulés, mais aussi deux variables supplémentaires, créées par la vibration des cordes vocales du larynx et par la résonance de la cavité nasale grâce à l'abaissement du voile du palais (velum). Dans notre classification, $L$ et $V$ désignent deux articulateurs supplémentaires (larynx et velum), responsables des processus du voisement et de la nasalisation respectivement. 0 représente l'absence de ces processus pour les phonèmes non voisés et oraux. En outre, comme nous l'avons indiqué plus haut, le phonème [ə] est

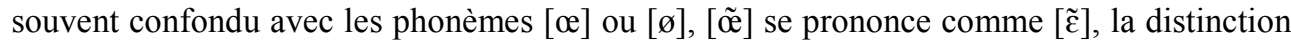
entre [a] et [a] tend aussi à s'atténuer, tout comme celles entre [o] et [o], entre $[\varepsilon]$ et [e]. En d'autres mots, selon Martinet [22] et Walter [39], ces oppositions phonologiques du

${ }^{2}$ Pour une discussion plus détaillée sur la différence entre ces semi-voyelles et les voyelles correspondantes, nous renvoyons à Martinet [24]. 
français sont neutralisées. Par conséquent, nous ne considérons pas ces phonèmes séparément, mais plutôt comme archiphonèmes dans le sens de Martinet (ibid.), c'est-à-dire comme la somme des caractéristiques pertinentes des phonèmes d'une opposition phonologique. Ces archiphonèmes sont notés par des lettres majuscules $([\mathrm{E}],[\mathrm{E}],[\tilde{\mathrm{E}}],[\mathrm{O}]$, [A]), dans le Tableau 2.

Le Tableau 2 parvient à illustrer une approche holistique de la classification des phonèmes du français standard. Tout comme pour les phonèmes d'occlusion, les propriétés des phonèmes d'aperture sont basées sur la position des articulateurs mobiles. Ainsi, l'opposition traditionnelle entre les voyelles "antérieures» et "postérieures » est remplacée par la position des parties pré-dorsale ou dorsale de la langue, et l'opposition entre les voyelles « ouvertes » et "fermées » se réfère à différents degrés d'aperture de la cavité buccale qui dépendent essentiellement de la position et des mouvements de la langue et des lèvres.

\section{Les particularités de la distribution des phonèmes}

Les données suivantes représentent l'analyse de la distribution des phonèmes en mots monosyllabiques $\mathrm{du}$ français standard. Nous considérons que cette distribution est le résultat de l'interaction, même du conflit, entre le facteur communicatif et le facteur humain. Tobin [30] appelle ce conflit la lutte mini-max de communication linguistique, qui consiste à atteindre le maximum de communication avec le minimum d'effort. Ainsi, nous interpréterons les données statistiques quant à la fréquence des phonèmes et montrerons que le facteur humain de "moindre effort» détermine la fréquence relativement faible des phonèmes qui nécessitent un plus grand degré de contrôle des muscles des organes phonateurs, tandis que le facteur communicatif justifie une augmentation d'effort pour la production de certains phonèmes au nom de la spécificité communicative. En outre, nous essaierons de trouver des justifications en dehors du domaine linguistique qui reflètent les caractéristiques psycho-physiologiques des locuteurs qui emploient la langue à des fins communicatives.

\subsection{Les apicales en position finale ont une fréquence plus élevée}

Si nous croyons que le contrôle des muscles des organes phonateurs est essentiel pour la production des phonèmes, il n'est pas surprenant que nous attachions une grande importance à la dextérité de la pointe de la langue (l'apex), un fait qui semble jouer un rôle considérable dans la distribution des sons apicaux. La pointe est une partie de la langue qui est la plus flexible, la plus sensible et la plus facile à contrôler de tous les articulateurs mobiles. Tel un droitier qui utilise sa main droite (plus habile et rapide) pour les tâches courantes et surtout pour celles qui nécessitent plus de précision (voir Diver [9]), les locuteurs peuvent employer des sons apicaux en positions dans le mot où un effort minimal peut être appliqué sans nuire à la communication. La position initiale est d'une extrême importance pour la communication car elle apporte la plus grande quantité d'information pour le locuteur et pour l'auditeur. En revanche, en position finale, l'importance de la communication est diminuée tant pour le locuteur que pour l'auditeur car la quantité d'information qui pourrait contribuer à l'identification du mot a déjà été fournie au début et au milieu d'où la nécessité des sons qui exigent peu d'effort musculaire. Diver (ibid.), Davis [6] et Tobin [31] montrent que les sons apicaux [t], [d], [s], [z], [n], [1] sont significativement préférés en position finale en anglais, en italien et en hébreu respectivement. Nous examinerons maintenant si la tendance d'employer les sons apicaux en position où le poids comminicatif est maximal et là où il est minimal se manifeste en français. Les résultats de ce calcul apparaissent dans le Tableau 3. 
Tableau 3. Les apicales en position finale ont une fréquence plus élevée

\begin{tabular}{lllll}
\hline & \multicolumn{2}{l}{ Articulateurs mobiles } & & \\
& Pointe & Lame & Lèvres & Dos \\
\cline { 2 - 5 } Position initiale & 1610 & 325 & 1976 & 1019 \\
Position finale & 2028 & 595 & 723 & 1119 \\
Rapport de diminution en finale & & $3.41: 1$ & $2.80: 1$ & $1.81: 1$ \\
\hline
\end{tabular}

Note $: \chi^{2}<0.0001$

Les données du Tableau 3 indiquent clairement que les sons apicaux sont très fréquents en position initiale où ils cèdent la première place aux sons labiaux pour une raison dont nous discuterons plus tard. En revanche, en position finale, où le poids communicatif est minimal, les sons apicaux sont trois fois plus fréquents que les sons pré-dorsaux (c'est-àdire prononcés avec la lame de la langue), environ trois fois plus fréquents que les sons labiaux (prononcés avec les lèvres) et environ deux fois plus fréquents que les sons dorsaux (c'est-à-dire prononcés avec le dos de la langue $)^{3}$.

\subsection{Les articulateurs supplémentaires réduisent la fréquence des phonèmes}

Supposons, à titre d'exemple, que quelqu'un veuille apprendre à conduire une voiture et hésite entre une voiture à boîte de vitesses mécanique et une à boîte automatique. La voiture à boîte mécanique, bien que moins coûteuse, a un inconvénient: il faut non seulement tenir et manœuvrer le volant et appuyer tantôt sur l'accélérateur, tantôt sur le frein, mais il faut le faire tout en changeant de vitesses à l'aide d'un levier et en appuyant sur la pédale d'embrayage, c'est-à-dire en effectuant des mouvements supplémentaires. Comme nous l'avons remarqué précédemment, la production de sons est une sorte de mouvement (ou plutôt de contraction) des muscles des organes phonateurs (langue, lèvres, voile du palais). Pour cette raison, le nombre d'articulateurs, impliqués dans la production des phonèmes, peut expliquer la distribution phonotactique dans le mot. Si la production d'un phonème nécessite l'intervention d'un articulateur supplémentaire, on doit s'attendre à une augmentation d'effort et, donc, à la fréquence relativement plus faible du phonème en question.

\subsubsection{Un articulateur (dévoisé, oral) a une fréquence plus élevée; deux articulateurs (voisés, nasaux) ont une fréquence plus faible}

Ce que nous avons dit ci-dessus s'applique au voisement des phonèmes et se manifeste de façon particulièrement évidente dans les oppositions entre les phonèmes d'occlusion dévoisés et voisés aussi bien que dans les oppositions entre les phonèmes d'aperture oraux et nasaux ${ }^{4}$. Le voisement met en jeu deux paires de muscles simultanément : la contraction des muscles du canal vocal est accompagnée d'une vibration des cordes vocales (voir Diver [9]). Ceci laisse supposer qu'un plus grand effort, dû à l'addition d'un articulateur, c'est-àdire des cordes vocales du larynx, réduira la fréquence des sons voisés. On pourrait

\footnotetext{
${ }^{3}$ On pourrait rétorquer que s'il y a plus de phonèmes apicaux, on s'attend à ce qu'ils soient globalement plus fréquents. Pourtant, affirmer qu'un phonème a une assez grande fréquence ne veut pas dire que cette fréquence soit nécessairement significative du point de vue statistique pour démontrer la validité d'une hypothèse.

${ }^{4}$ En ce qui concerne les phonèmes d'occlusion, dits «sonantes», ou les phonèmes d'aperture oraux qui sont tous sonores en français, on augmente un effort pour leur production afin d'être clairement perçu dans la communication. En revanche, des sonantes ou des voyelles sourdes ont un rendement communicatif très faible ce qui explique leur absence dans la plupart des langues, y compris le français.
} 
s'attendre à ce que les phonèmes voisés $([\mathrm{b}],[\mathrm{v}],[\mathrm{d}],[\mathrm{z}],[3],[\mathrm{g}])$ aient une fréquence inférieure à celle des phonèmes non voisés $([\mathrm{p}],[\mathrm{f}],[\mathrm{t}],[\mathrm{s}],[\mathrm{f}],[\mathrm{k}])$. Le rapport entre ces phonèmes est résumé dans le Tableau 4 suivant.

Tableau 4. Les phonèmes d'occlusion en positions initiale et finale. Un articulateur (dévoisé) a une fréquence plus élevée; deux articulateurs (voisés) ont une fréquence plus faible.

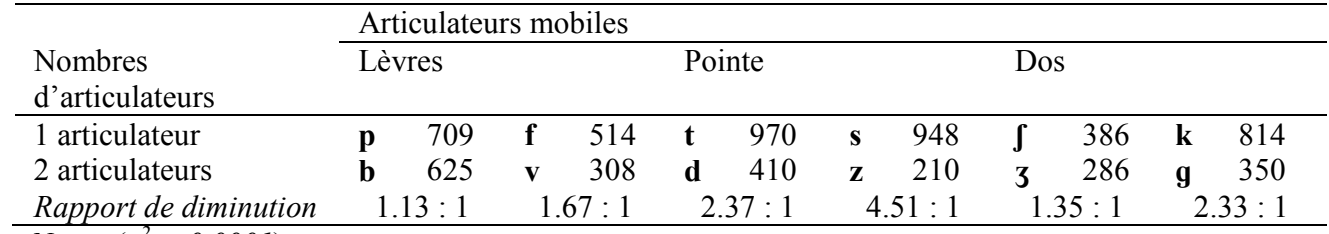

Note : $\left(\chi^{2}<0.0001\right)$

Comme on le voit dans le Tableau 4, les phonèmes non voisés, produits par un articulateur, ont une fréquence supérieure à celle des phonèmes voisés, produits par deux articulateurs à la fois.

La différence peu profonde que celle qu'il y a entre la fréquence des non voisées [p] et [f], d'une part, et des voisées [b] et [v], de l'autre, peut s'expliquer par le facteur communicatif. Nous y reviendrons plus loin.

La fréquence analogue du phonème non voisé [ $\left.\int\right]$ et du phonème voisé [3] peut être due aux nombreuses origines étymologiques de celui-ci (voir Brachet [3], Nyrop [27]). Ce phonème dérive tantôt de la palatale [j], comme dans le mot jeune [3oen] (du latin vulgaire jŏvenis, altéré du latin classique jǔvenis), tantôt de la vélaire [g], comme dans jouir [3wi:r] (du latin vulgaire gaudire, altéré du latin classique gaudere), tantôt de la voyelle [e], comme dans je [3ə] (du latin vulgaire eo, altéré du latin classique ego). Son équivalent dévoisé [S] provient principalement de la vélaire [k], suivie de la voyelle [a] (voir Brachet [ibid.], Nyrop [ibid.]), comme dans chef [ $\mathrm{ff}]$ (du latin vulgaire capum, altéré du latin classique caput).

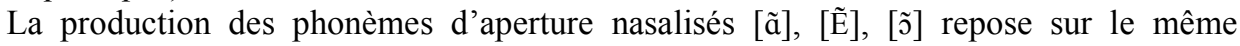
principe de l'addition d'un articulateur que celui des phonèmes d'occlusion voisés: l'abaissement du voile du palais, permettant l'écoulement de l'air par la cavité nasale, fait ajouter la résonance du nez à celle de la bouche. Il est donc légitime de supposer que la combinaison de deux articulations concomitantes, orale et nasale, réduira la fréquence des sons nasaux. Les données statistiques sont réunies dans le Tableau 5 suivant.

Tableau 5. Les phonèmes d'aperture. Un articulateur (oral) a une fréquence plus élevée ; deux articulateurs (nasaux) ont une fréquence plus faible.

\begin{tabular}{llllrlr}
\hline & \multicolumn{3}{l}{ Articulateurs mobiles } & & & \\
\cline { 2 - 7 } Nombres d'articulateurs & Lame / Dos & Lame & & \multicolumn{2}{l}{ Lèvres + Dos } \\
\hline 1 articulateur & $\mathbf{A}$ & 1207 & $\mathbf{E}$ & 913 & $\mathbf{O}$ & 769 \\
2 articulateurs & $\tilde{\mathbf{a}}$ & 316 & $\tilde{\mathbf{E}}$ & 212 & $\tilde{\mathbf{j}}$ & 194 \\
Rapport de diminution & & $3.82: 1$ & & $4.31: 1$ & & $3.96: 1$ \\
\hline
\end{tabular}

Note : $\left(\chi^{2}<0.0001\right)$

Ces données confirment notre hypothèse : on peut voir la diminution considérable des phonèmes d'aperture nasaux par rapport aux phonèmes oraux.

\subsubsection{Trois articulateurs (voisement et nasalisation) ont une fréquence moindre}

La production des phonèmes d'occlusion nasalisés $[\mathrm{m}],[\mathrm{n}],[\mathrm{n}],[\mathrm{y}]$ ressemble à celle des phonèmes d'aperture nasalisés. Elle implique la participation des organes mobiles (les lèvres, la pointe de la langue ou le dos de la langue) avec la vibration des cordes vocales, 
accompagnée du passage de l'air par la cavité nasale, comme résultat de l'abaissement du voile du palais lors de l'articulation, c'est-à-dire la combinaison de trois articulations concomitantes. Par conséquent, « on pourrait s'attendre à ce que les usagers favorisent inconsciemment l'emploi » (Martinet [23]) des phonèmes voisés «et restreignent plus ou moins l'emploi » (ibid.) des phonèmes nasalisés. Le Tableau 6 montre l'écart entre les phonèmes d'occlusion en position initiale, en fonction du nombre d'articulateurs.

Tableau 6. Les phonèmes d'occlusion en positions initiale et finale. Un articulateur (dévoisé) a une fréquence plus élevée ; deux articulateurs (voisés) ont une fréquence plus faible ; trois articulateurs (voisement et nasalisation) réduisent encore davantage la fréquence

\begin{tabular}{lllllll}
\hline Nombre d'articulateurs & \multicolumn{2}{l}{ Articulateurs mobiles } & & & \\
\cline { 2 - 7 } (\& de difficultés qui se multiplient) & Lèvres & Pointe & \multicolumn{3}{l}{ Dos } & \\
\hline 1 articulateur & $\mathbf{p}$ & 709 & $\mathbf{t}$ & 970 & $\mathbf{k}$ & 814 \\
2 articulateurs & $\mathbf{b}$ & 625 & $\mathbf{d}$ & 410 & $\mathbf{g}$ & 350 \\
3 articulateurs & $\mathbf{m}$ & 543 & $\mathbf{n}$ & 382 & $\mathbf{n}$ & 3 \\
\% de diminution de 3 articulateurs & & $56.6 \%$ & & $71.7 \%$ & & $99.6 \%$ \\
\hline
\end{tabular}

Note $:\left(\chi^{2}<0.0001\right)$

Dans le Tableau 6, nous pouvons observer la diminution du nombre de mots en raison du nombre d'articulateurs : du voisement ( 2 articulateurs) à la nasalisation (3 articulateurs). On constate que la fréquence diminue à mesure que les articulateurs et les difficultés se multiplient. Pour les labiales, il y a une diminution de 166 mots, soit 56.6\%, de 709 mots qui commencent ou finissent par [p] (un articulateur) à 543 mots qui commencent ou finissent par [m] (trois articulateurs). Pour les apicales, il y a une diminution de 588 mots, c'est-à-dire $71.7 \%$, de 970 mots qui commencent ou finissent par [t] (un articulateur) à 382 mots qui commencent ou finissent par [n] (trois articulateurs). En outre, ces données montrent que plus on arrive à l'arrière de la cavité buccale, plus l'emploi des nasales se restreint jusqu'à l'absence complète de la dorsale [y] en position initiale, ne laissant que trois occurrences en position finale. Cela n'est pas surprenant, car, en général, les articulateurs en arrière de l'espace buccal sont plus difficiles à contrôler. Le même phénomène s'observe dans les phonèmes d'aperture : le nombre de mots monosyllabiques, contenant les phonèmes dits " antérieurs », c'est-à-dire ceux qui sont produits avec la partie avant de la langue, s'élève à 3382 , soit à $64 \%$ de tous les mots monosyllabiques en français standard. Martinet, cité par Peeters [28], s'est exprimé en ces termes à ce sujet :

«Dès le début, Martinet (1939d: 285) a fait remarquer que «par suite de la conformation des organes, la série postérieure, articulée plus près de la charnière de la bouche, a moins de latitude que la série antérieure ». En d'autres termes (Martinet 1958 : $351=1969$ : 200), «l'espace disponible est moindre à l'arrière ». A la suite de cette différence de latitude, " un parallélisme rigoureux entre les deux séries a souvent de la peine à se maintenir » (Martinet 1939d: 285), et cèdera le pas devant la tendance à l'équidistance. Il y aura plus de phonèmes antérieurs que de phonèmes postérieurs [...]. »

\subsection{Les phonèmes "visibles " ont une fréquence plus élevée en position initiale}

Dans son analyse, Diver [9] soutient que la position initiale du mot a une charge communicative plus considérable. Davis [6] adopte cette idée et montre qu'en italien en position initiale il y a une légère préférence pour les labiales, produites avec les deux lèvres, c'est-à-dire qui sont «visibles ». Il reste à examiner si le même phénomène se retrouve également en français. La préférence pour les phonèmes d'occlusion labialisés en position initiale est présentée dans le Tableau 7. 
Tableau 7. Les articulations « visibles » ont une fréquence plus élevée en position initiale

\begin{tabular}{lcccccc}
\hline Articulateurs mobiles & \multicolumn{1}{c}{} & & & \\
\cline { 2 - 7 } Lèvres (sons « visibles ») & $\mathbf{p}$ & 531 & $\mathbf{b}$ & 527 & $\mathbf{m}$ & 328 \\
Pointe & $\mathbf{t}$ & 412 & $\mathbf{d}$ & 206 & $\mathbf{n}$ & 142 \\
\% de diminution & $22.4 \%$ & & $60.9 \%$ & & $56.7 \%$ \\
\hline
\end{tabular}

Note : $\left(\chi^{2}<0.0001\right)$

Il ressort clairement du Tableau 7 que les labiales (les phonèmes «visibles ») ont une fréquence plus élevée en position initiale que les apicales. Dans chacune des paires de phonèmes, le nombre d'articulateurs reste le même (les non voisés [p] et [t] sont produits avec un articulateur, les voisées [b] et [d] - avec deux, les nasalisés [m] et [n] - avec trois), mais on change d'articulateur. Les données montrent que dans les trois paires, les phonèmes labialisés ( «visibles ») sont plus fréquents que leurs équivalents non labialisés. On peut voir la diminution de la fréquence des phonèmes, produits avec un articulateur : de 531 mots qui commencent par [p] labialisé ("visible ») à 412 mots qui commencent par l'apicale [t], c'est-à-dire une diminution de $22.4 \%$. Les phonèmes, produits avec deux et trois articulateurs, ont une diminution de $60.9 \%$ et de $56.7 \%$ respectivement. Cela n'est pas étonnant, car les phonèmes labialisés peuvent être perceptibles à la fois par la vue et par l'ouïe (voir Tobin [31]). Ils fournissent un plus grand nombre d'indices, rendant ainsi la communication plus facile et plus efficace.

\section{Résumé et conclusions}

Pour résumer, dans cet article, nous avons appliqué la théorie de la $\mathrm{PCH}$ à la langue française. Suivant Diver [9], Davis [6] et Tobin [31], nous avons remplacé des catégories phonétiques et phonologiques traditionnelles par des catégories alternatives qui sont plus orientées vers la physiologie, la perception, la cognition et le comportement humains et qui comprennent :

- la hiérarchie unique des phonèmes basée sur certains degrés d'aperture et sur des types d'écoulement de l'air, par opposition aux catégories traditionnelles de consonnes et de voyelles et différentes propriétés traditionnelles, liées au mode d'articulation ;

- les articulateurs mobiles à l'aide desquels les phonèmes sont produits, par opposition au concept traditionnel de « lieu d'articulation » qui est associé aux récepteurs passifs ;

- le nombre d'articulateurs nécessaires pour la production des phonèmes, par opposition aux concepts traditionnels de sons « non voisés / voisés / nasalisés ».

Nous avons montré que certains aspects de la distribution des phonèmes peut être expliquée par le principe de lutte mini-max qui «représente la synthèse des besoins et de l'inertie » (Martinet [23]), c'est-à-dire le conflit entre le facteur communicatif et le facteur humain dans la recherche du maximum de communication avec le minimum d'effort (voir Tobin [30]). Tout au long de cette analyse, nous avons examiné une corrélation entre l'effort déployé par les locuteurs pour contrôler les articulateurs mobiles, impliqués dans la production des phonèmes, et la fréquence observée de ces phonèmes dans différentes distributions phonotactiques. Les principes suivants ont été confirmés :

1. La fréquence des apicales est particulièrement élevée en position finale.

2. Les articulateurs supplémentaires réduisent la fréquence des phonèmes, sauf si leur utilisation sert des fins communicatives.

3. Les phonèmes « visibles » ont une fréquence plus élevée en position initiale.

Nous avons essayé de montrer que les suites de mouvements qui sont plus faciles à articuler et, par conséquent, plus faciles à apprendre à contrôler sont généralement préférées à celles qui exigent une augmentation d'effort et, donc, qui sont plus difficiles à produire et 
plus difficiles à apprendre à contrôler, sauf si leur utilisation fournit plus d'informations acoustiques, permettant de créer plus d'oppositions distinctes.

Nous tenons à remercier Yishai Tobin pour ses suggestions très précieuses. Nous sommes seuls responsables des insuffisances et des erreurs qui pourraient s'introduire.

\section{Références}

1. S. Anderson. The analysis of French schwa: or how to get something for nothing, Language 58, 534-573 (1982).

2. A. Azim. Problems of Aspiration in Modern Standard Urdu, in: W. Reid, R. Otheguy, N. Stern (eds.) Signal, Meaning, and Message, 273-307 (Amsterdam/Philadelphia, Benjamins, 2002).

3. A. Brachet. Grammaire historique de la langue française (Littré, J. Hetzel, 1880).

4. F. Carton. Introduction à la phonétique du français (Paris/Bruxelles/Montréal, Bordas, 1974).

5. J. Casagrande. The Sound System of French (Washington, Georgetown University Press, 1984).

6. J. Davis. A Combinatory Phonology of Italian, Part I: Initial constriction to maximal aperture, in: CUWPL 8, 1-99 (1984 [1987]).

7. P. Delattre. La question des deux «a » en français, The French Review, 31.2, 141-148 (1957).

8. N. Delbecque (éd.) Linguistique cognitive: Comprendre comment fonctionne le langage (Bruxelles, De Boeck Supérieur, 2006).

9. W. Diver. Phonology as Human Behavior, in: D. Aaronson, R.W. Rieber (eds.) Psycholinguistic Research: Implications and applications, 161-186 (Hillsdale, NJ, Lawrence Erlbaum, 1979).

10. W. Diver. The Phonology of the Extremes. (Third International Columbia School Conference on Linguistics. Rutgers University, New Brunswick, NJ, 1993).

11. W. Diver. Theory, in: E. Contini-Morava, B. Sussman Goldberg (eds.) Meaning as Explanation: Advances in linguistic sign theory, 43-114 (Berlin, Mouton de Gruyter, 1995).

12. I. Dreer. Phonology as human behavior: a combinatory phonology of Byelorussian, in:

J. Davis, R.J. Gorup, N. Stern (eds.), Advances in functional linguistics: Columbia School beyond its origins, 107-130 (Amsterdam/Philadelphia, Benjamins, 2006).

13. I. Dreer. Expressing the Same by the Different (Amsterdam/Philadelphia, Benjamins, 2007).

14. Z. Fagyal, D. Kibbee, F. Jenkins. French: A Linguistic Introduction (Cambridge, Cambridge University Press, 2006).

15. A.R. Fatihi. Economy of Articulation in Mewati Phonology (Aligarh Muslim University, U.P., India, 1987).

16. N. Flores. The Distribution of Post-vocalic Phonological Units in Spanish. (Fifth International Columbia School Conference on Linguistics, Rutgers University, New Brunswick, NJ, 1997).

17. R. S. Gess, Ch. Lyche, T. Meisenburg. Introduction to phonological variation in French: Illustrations from three continents, in: R. S. Gess, Ch. Lyche, T. Meisenburg (eds.), Phonological Variation in French: Illustrations from Three Continents, 1-19 (Amsterdam/Philadelphia, Benjamins, 2012). 
18. A. Hansen. Étude du E caduc - stabilisation en cours et variations lexicales, Journal of French Language Studies 4, 25-54 (1994).

19. Sh.Sh. Jabeen. Economy of Articulation in the Phonology of Bihar Urdu. (Aligarh Muslim University, U.P., India, 1993).

20. P.R. Léon. Apparition, maintien et chute du 'E caduc', La Linguistique 2, 111-122 (1966).

21. A.R. Lodge, N. Armstrong, Y.M.L. Ellis, J.F. Shelton. Exploring the French Language (London, Hodder Arnold, 1997 [2003]).

22. A. Martinet. Phonology as functional phonetics. Les traits généraux de la phonologie du français (Oxford, Blackwell, 1949 [1955]).

23. A. Martinet. Économie des changements phonétiques (Berne, Éditions A. Francke, 1955 [1970]).

24. A. Martinet. Remarques sur le système phonologique du français, in : Bulletin de la Société de Linguistique de Paris 34, 191-202 (Paris, Librairie C. Klincksieck, 1933).

25. Y.-C. Morin. De l'ajustement du schwa en syllabe fermée dans la phonologie du français, in: S.P. Verluyten (éd.) La phonologie $d u$ schwa français, 133-189 (Amsterdam, Benjamins, 1988).

26. Y.-C. Morin. Le français de référence et les normes de prononciation, Cahiers de l'Institut de Linguistique de Louvain 26, 91-135 (2000).

27. K. Nyrop. Grammaire historique de la langue française. (Paris, Alphonse Picard \& fils, 1899).

28. B. Peeters. Diachronie, phonologie, et linguistique fonctionnelle (Louvain-la-Neuve, Peeters, 1992).

29. F. de Saussure. Cours de Linguistique Générale (Paris, Payot, 1916 [2001]).

30. Y. Tobin. Invariance, markedness and distinctive feature analysis: a contrastive study of sign systems in English and Hebrew (Amsterdam/Philadelphia, Benjamins, 1995).

31. Y. Tobin. Phonology as Human Behavior (Durham, NC, Duke University Press, 1997).

32. Y. Tobin. Developmental and Clinical Phonology: The Prague School and beyond, in: Travaux du Cercle Linguistique de Prague 3, 53-68 (1999).

33. Y. Tobin. Comparing and Contrasting Optimality Theory with the Theory of Phonology as Human Behavior, in: Linguistic Review 17(2-4), 291-301 (2000).

34. Y. Tobin. Phonology as Human Behavior: Initial consonant clusters across languages, in: W. Reid, R. Otheguy, N. Stern. (eds.) Signal, Meaning, and Message, 191-255 (Amsterdam/Philadelphia, Benjamins, 2002).

35. B. Tranel. The Sounds of French: An Introduction (Cambridge, Cambridge University Press, 1987 [1998]).

36. B. Tranel. À propos de l'ajustement de E en français, in : S.P. Verluyten (éd.) $L a$ Phonologie du schwa français, 89-131 (Amsterdam, Benjamins, 1988).

37. D.C. Walker. The new stability of unstable -e in French, Journal of French Language Studies 6.2, 211-229 (1996).

38. D.C. Walker. French Sound Structure (Calgary, University of Calgary Press, 2001).

39. H. Walter. La phonologie du français (Paris, Presses Universitaires de France, 1977). 